\title{
Mutations in the glucocorticoid receptor zinc finger region that distinguish interdigitated DNA binding and transcriptional enhancement activities
}

\author{
Mark Schena, Leonard P. Freedman, and Keith R. Yamamoto \\ Department of Biochemistry and Biophysics, University at California-San Francisco, San Francisco, California 94143-0448 \\ USA
}

\begin{abstract}
Mammalian glucocorticoid receptors bind specifically to glucocorticoid response element (GRE) DNA sequences and enhance transcription from GRE-linked promoters in mammalian cells and in yeast. We randomly mutagenized a segment of the receptor encompassing sequences responsible for DNA-binding and transcriptional regulation and screened in yeast for receptor defects. The mutations all mapped to a 66-aminoacid subregion that includes two zinc fingers; in general parallel phenotypes were observed in yeast and animal cells. Mutants defective for DNA binding also failed either to enhance or to repress transcription. However, several mutations in the second finger selectively impaired enhancement; we suggest that such 'positive control' mutants may alter protein-protein contacts required for transcriptional activation.
\end{abstract}

[Key Words: GRE; zinc finger domain; DNA binding; transcription]

Received July 5, 1989; revised version accepted August 14, 1989.

In the presence of bound hormone, the glucocorticoid receptor protein regulates transcription initiation from specific animal cell promoters used by RNA polymerase II. The receptor enhances transcription by associating selectively with DNA sequences termed glucocorticoid response elements (GREs) (Chandler et al. 1983; Payvar et al. 1983; Scheidereit et al. 1983). In addition, the receptor represses transcription by binding to a distinct class of sequences termed negative GREs (nGREs) (Sakai et al. 1988). In association with the receptor, both GREs and nGREs act 'at a distance' to modulate the activity of linked promoters, thereby serving as enhancers and long-range operators, respectively (Chandler et al. 1983; Sakai et al. 1988).

Functional regions of the glucocorticoid receptor and other members of the nuclear receptor gene superfamily have been characterized in some detail (Evans 1988; Green and Chambon 1988; Beato 1989). Studies of the 795-amino-acid rat glucocorticoid receptor demonstrated, for example, that a 150-amino-acid fragment, residues 407-556, is sufficient for GRE and nGRE binding (Rusconi and Yamamoto 1987; Mordacq and Linzer 1989), nuclear localization (Picard and Yamamoto 1987), transcriptional enhancement (Miesfeld et al. 1987; Godowski et al. 1988|, and transcriptional repression (Miesfeld et al. 1988). This segment of the receptor includes a 61-amino-acid subregion containing two zinc finger motifs (Miller et al. 1985; Weinberger et al. 1985; Berg 1986); Freedman et al. (1988) showed that the segment indeed binds two zinc ions, each coordinated te- trahedrally by four cysteine sulfur atoms, and that metal coordination is essential for proper folding and DNA binding. In addition, a 28-amino-acid subfragment has been identified, which lies downstream of the fingers, that exhibits nuclear localization activity but fails to bind DNA or to modulate transcription (Picard and Yamamoto 1987; Rusconi and Yamamoto 1987). Thus, of the four activities resident within the $407-556$ region (denoted here as the finger domain), only nuclear localization has been uncoupled from the others.

What is the relationship of the DNA-binding and transcriptional regulatory activities within the finger domain? A commonly held view is that long-range regulators such as the receptor bind to DNA sequences in the vicinity of promoters that they regulate and then confer regulation via protein-protein interactions with a component of the transcription initiation machinery (Ptashne 1986, 1988; Yamamoto 1985, 1989). According to this view, DNA binding is essential for regulation, and the individual residues that mediate DNA binding and regulation are, at least in part, distinct; this has been shown clearly to be the case for proteins containing regulatory domains that are widely separated from their cognate DNA-binding domains (Hope and Struhl 1986; Ma and Ptashne 1987; Godowski et al. 1988).

To pursue the functional complexity of the finger domain, we sought to develop a rapid and efficient genetic approach. Recently, it has been demonstrated that various transcriptional regulators and initiation factors can function in both yeast and mammalian cells /Cho- 
dosh et al. 1988; Kakidani and Ptashne 1988; Lech et al. 1988; Struhl 1988; Webster et al. 1988;). In particular, the glucocorticoid receptor (Schena and Yamamoto 1988) and the estrogen receptor (Metzger et al. 1988) confer transcriptional enhancement on promoters in yeast that are linked to GREs and estrogen response elements (EREs), respectively. These findings suggested that we should be able to exploit the genetic capabilities of yeast to screen a large number of random point mutations within the glucocorticoid receptor as a step toward developing a broad genetic approach to dissecting functional receptor domains and identifying interacting cellular factors. Therefore, we mutagenized a fragment of receptor cDNA encoding the finger domain, inserted those segments into a wild-type receptor recipient, and screened in yeast for mutants defective in the expression of a GRE-linked reporter gene. We report here the isolation and preliminary characterization, in yeast, in animal cells, and in vitro, of a series of such point mutations.

\section{Results}

\section{Mutagenesis and screening}

To isolate mutants defective in the DNA-binding and transcriptional regulatory functions of the receptor finger domain, we employed random mutagenesis coupled with a genetic screen in yeast (Fig. 1). A 430-bp XhoI-Sst fragment that encodes 143 amino acids (residues 414-556) encompassing the finger domain was mutagenized in vitro with sodium nitrite (see Experimental procedures). Then the fragments were reinserted into an unmutagenized receptor expression vector cleaved with XhoI and Sst I to generate a pool of $10^{4} \mathrm{de}$ rivatives bearing a high frequency of point mutations within the 414-556 region. To simplify our manipulations, the recipient vector plasmid encoded N556a, a truncated receptor derivative that lacks the hormonebinding domain, amino acids 557-795 (Rusconi and Yamamoto 1987), and is constitutively active in animal cells (Godowski et al. 1987) and in yeast (Schena and Yamamoto 1988). The mutagenized plasmids were transformed into a yeast strain containing an integrated, GRE-linked Escherichia coli $\beta$-galactosidase (lacZ) reporter gene driven by the yeast CYC1 promoter (Guarente and Hoar 1984). Colonies expressing wild-type N556a are dark blue on X-Gal indicator plates under these conditions. White colonies (putative $l a c Z^{-}$) appeared at a rate of $\sim 4 \times 10^{-3}$, and light blue colonies (reduced lacZ expression) were observed at $\sim 4 \times 10^{-4}$. Fifty colonies displaying these phenotypes were isolated and subcloned; those defective in $\beta$-galactosidase expression were commonly larger than those expressing normal levels, consistent with the finding that functional receptor derivatives reduce the growth rate of yeast slightly (M. Schena, unpubl.).

\section{Sequence alterations and protein stability of the mutants}

The receptor expression plasmids from 50 yeast clones deficient in $\beta$-galactosidase expression were transformed into E. coli for efficient propagation, and the mutagenized segments were sequenced. Single, double, or triple point mutations (in a ratio of $5: 2: 1$ ) were detected in 41 plasmids, and all but three of the base changes were $\mathrm{G}$ to $\mathrm{A}$ or $\mathrm{T}$ to $\mathrm{C}$ transitions, as expected for sodium nitrite mutagenesis (Myers et al. 1985). The remaining nine plasmids lacked mutations and exhibited normal $\beta$-galactosidase production upon retesting in yeast, suggesting that they were false positives in the initial screen. The lesions within the 25 plasmids that carried single point mutations were all clustered in a 66-aminoacid segment (residues 440-505) that corresponds closely to the 61 amino acids proposed to form the two zinc fingers (Fig. 2A). Moreover, of the 16 plasmids containing double or triple mutations, each contained at least one alteration within the 66-amino-acid region. The defects in 10 such multiple mutants were separated to generate 14 additional single mutants and 1 double

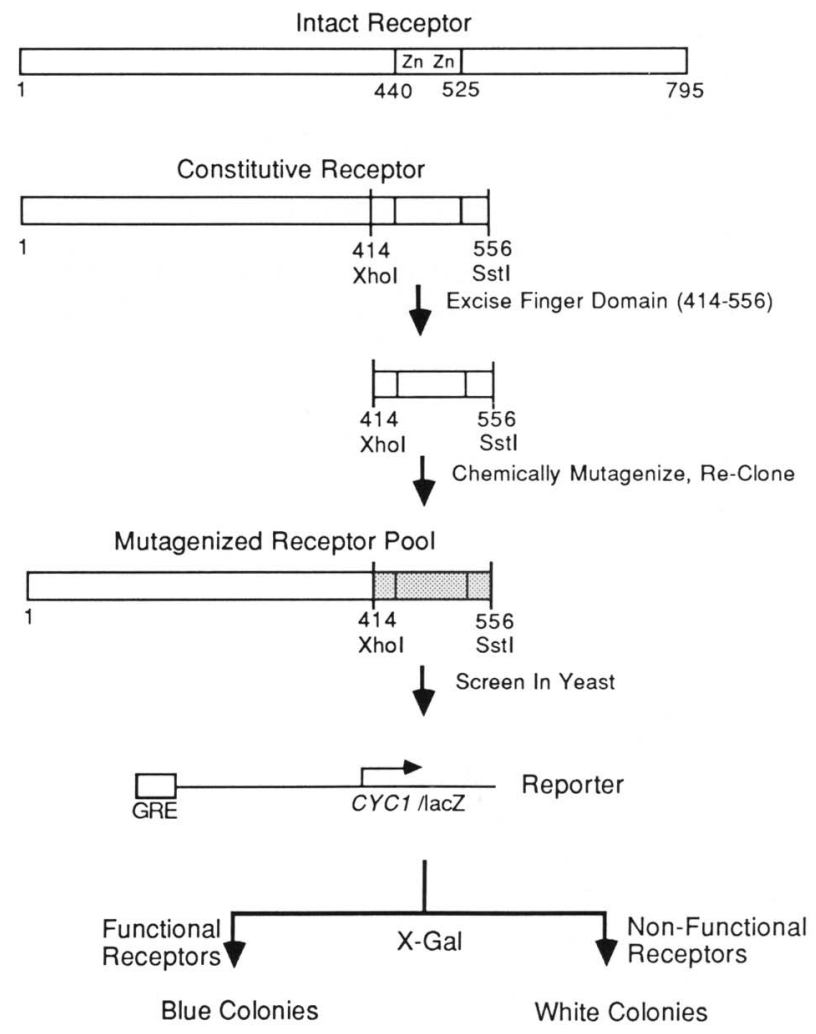

Figure 1. Mutagenesis and screening of receptor derivatives with defects in the zinc finger domain. A 430-bp XhoI-Sst I fragment was excised from a cDNA encoding a constitutive glucocorticoid receptor derivative, N556; the fragment, which encompasses the zinc finger region, was transferred to a singlestrand vector and mutagenized with sodium nitrite (see Experimental procedures). Mutagenized inserts were reinserted into the wild-type receptor backbone in a yeast expression plasmid, and these species were transformed into yeast strain BJ-G26.1, which bears an integrated GRE-linked reporter gene (Schena and Yamamoto 1988) consisting of the yeast CYC1 promoter fused to lacZ (Guarente and Hoar 1984). Yeast transformants expressing low levels of $\beta$-galactosidase were detected as white or light blue colonies on indicator plates. 


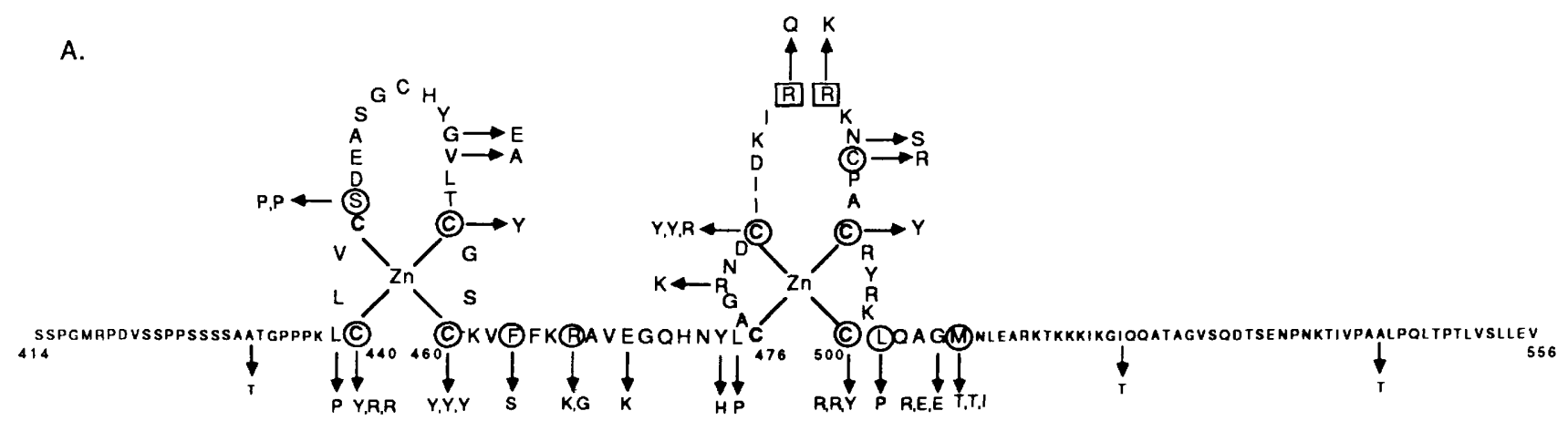

B.

$\begin{array}{lc}\text { Receptor } & \text { B.Gal(Unita) } \\ \text { Vector } & 2.7 \\ \text { Wild-Type (N556) } & 695 \\ \text { A432T } & 565 \\ \text { L439P } & 568 \\ \text { C440Y } & 2.4 \\ \text { C440R } & 2.3 \\ \text { S444P } & 4.0 \\ \text { G453E } & 440 \\ \text { V454A } & 563 \\ \text { C457Y } & 2.9 \\ \text { C460Y } & 2.9 \\ \text { F463S } & 2.2 \\ \text { R466K } & 2.6 \\ \text { R466G } & 3.6 \\ \text { E469K/Y474H } & 557 \\ \text { L475P } & 568\end{array}$

$\begin{array}{cc}\text { Receptor } & \text { B-Gal(Unite) } \\ \text { R479K } & 656 \\ \text { C482Y } & 2.5 \\ \text { C482R } & 2.0 \\ \text { R488O } & 19 \\ \text { R489K } & 70 \\ \text { N491S } & 404 \\ \text { C492R } & 2.5 \\ \text { C495Y } & 1.8 \\ \text { C500Y } & 3.5 \\ \text { C500R } & 2.6 \\ \text { L501P } & 3.0 \\ \text { G504R } & 580 \\ \text { G504E } & 577 \\ \text { M505T } & 3.7 \\ \text { M505I } & 3.5 \\ \text { I519T } & 497 \\ \text { A542T } & 348\end{array}$

Figure 2. Point mutations within the receptor finger domain. $(A)$ Receptor residues $414-556$ are depicted as two zinc fingers. Enlarged segment shows a 66-amino-acid subregion including all point mutations isolated within this region that impair receptor function. Circled residues indicate point mutations that each abolish receptor function in yeast; residues enclosed by squares indicate amino acid changes that partially impair receptor activity. Amino acids marked only with an arrow indicate neutral alterations with little or no phenotypic effect in yeast. Multiple isolates were obtained at some positions, as shown; in most cases, however, we could not determine whether multiple isolates of the same mutation represented independent mutational events. $(B \mid$ Each point mutant shown in $A$ was assayed for $\beta$-galactosidase activity, as described in Experimental procedures. Nomenclature for mutant receptor species includes the single-letter amino acid designation for a wild-type residue, followed by its position within the protein, and the single-letter code for the mutant residue; thus, L439P contains a leucine-to-proline substitution at receptor residue 439 . As a negative control, the same strain was transformed with an expression plasmid lacking receptor sequences (vector). All values represent the average of at least three independent measurements; individual determinations varied by $<20 \%$.

mutant. This revealed a series of 'neutral' mutations (e.g., N491S; see Fig. 2B)-amino acid alterations that did not impair significantly receptor function in our assays. Notably, the neutral mutations were distributed throughout the mutagenized fragment (e.g., A542T; see Fig. 2B). For each of the 10 multiple mutants, however, the mutant phenotype could be attributed to a lesion within the 66-amino-acid segment.

The double mutant and 38 of the single mutants were individually reintroduced into the yeast strain harboring a GRE-linked reporter plasmid; together, these represent 32 amino acid substitutions at 26 positions within the mutagenized fragment. (Mutant receptors are named by the single-letter designation of a wild-type amino acid and its position within the receptor, followed by the identity of the mutant residue; thus, C440Y contains a tyrosine substitution for cysteine at position 440 .)
Quantitative $\beta$-galactosidase measurements (Fig. 2B) confirmed the phenotypes ascribed originally by screening. We conclude that residues within and very close to the receptor zinc fingers are essential for GREmediated enhancement in yeast; in contrast, point mutations in the flanking residues (amino acids 414-439 and 506-556) are apparently not sufficient to alter enhancement activity. Mutations that abolished enhancement completely (Fig. 2A, circled residues) were located, in particular at cysteines proposed to coordinate zinc ions and at residues immediately carboxy-terminal to each finger. Mutations in the 'coordinating cysteines' likely alter the structure of the fingers (Freedman et al. 1988), whereas the lesions carboxy-terminal to the fingers may directly impair a receptor function, such as DNA recognition (e.g., see Mader et al. 1989).

We recovered several mutations that affected en- 
hancement but did not reside at the coordinating cysteines (see Discussion) or at the carboxy side of a finger. For example, S444P and C492R were fully defective. In addition, enhancement activity was reduced 10- to 30fold by mutations in either of the arginine residues at the 'tip' of the second finger (Fig. 2A, residues enclosed in squares; Fig. 2B). Consistent with these partial defects, R488Q and R489K were initially isolated as light blue colonies on indicator plates. Although the mutations at these positions cannot be interpreted without protein structural information, it is notable that these lesions also reside within the 66-amino-acid segment encompassing the zinc fingers. In contrast, neutral mutations mapped throughout the 143-amino-acid mutagenized receptor segment (Fig. 2A, residues marked only with arrows; Fig. 2B).

The observed mutant phenotypes may simply have reflected low-level expression or rapid degradation of the altered receptor derivatives. We therefore examined each of the mutants by immunoblotting of yeast extracts with a receptor-specific monoclonal antibody. The expression, solubility, and integrity of the mutant receptors were similar to that of the wild-type N556a receptor derivative in every case (Fig. 3, cf. lanes 4-20 with lane 3|. Thus, the failure of a mutant receptor to activate the GRE-linked reporter gene in yeast cells in no case reflected receptor underproduction or instability.

\section{DNA binding}

Freedman et al. (1988) used a bacteriophage T7 promoter and T7 polymerase system (Studier and Moffatt 1986) to overproduce and purify the receptor derivative X556 from E. coli; we adopted this same approach to overproduce the mutant X556 derivatives for DNA-binding studies (see Experimental procedures). Following expression and partial purification, wild-type and mutant receptor derivatives were tested for specific DNA binding in a gel retardation assay. With the wild-type X556 product, we detected two discrete retarded bands (complexes 1 and 2), which correspond to the binding of one and two receptors, respectively, to the labeled GRE-containing DNA fragment tested (J. La Baer and K.R. Yamamoto, unpubl.) (Fig. 4A, lanes 1 and 2). No binding was detected with 13 mutants that were fully defective for enhancement (Fig. 4A, lanes 3-11 and 15-20); titration experiments (data not shown) imply that GRE binding by these mutants is reduced by at least 50 -fold, relative to that of wild-type N556a. In contrast to these results, R $489 \mathrm{~K}$, which confers $\sim 10 \%$ of wild-type enhancement, displayed readily detectable DNA binding (Fig. 4A, lane 13); titration experiments indicated a 10-fold reduced affinity for the GRE-containing DNA fragment (data not shown). In addition, two mutants, R488Q and N491S, exhibited normal DNA binding (Fig. 4, cf. lanes 12 and 14 with lane 2). Interestingly, N491S is nearly fully active in yeast with respect to enhancement, whereas $\mathrm{R} 488 \mathrm{Q}$ produces $<3 \%$ the enhancement activity of N556a (Fig. 2B; see also Table 2, and Fig. 6).

An immunoblot of the protein fractions used for the DNA-binding experiments confirmed that the expected $19-\mathrm{kD}$ X556a receptor derivatives were produced at similar levels after induction of the appropriate bacterial cultures and that degradation was negligible (Fig. 4B); thus, differences in the in vitro DNA-binding properties of the various receptor mutants do not reflect differential expression or stability.

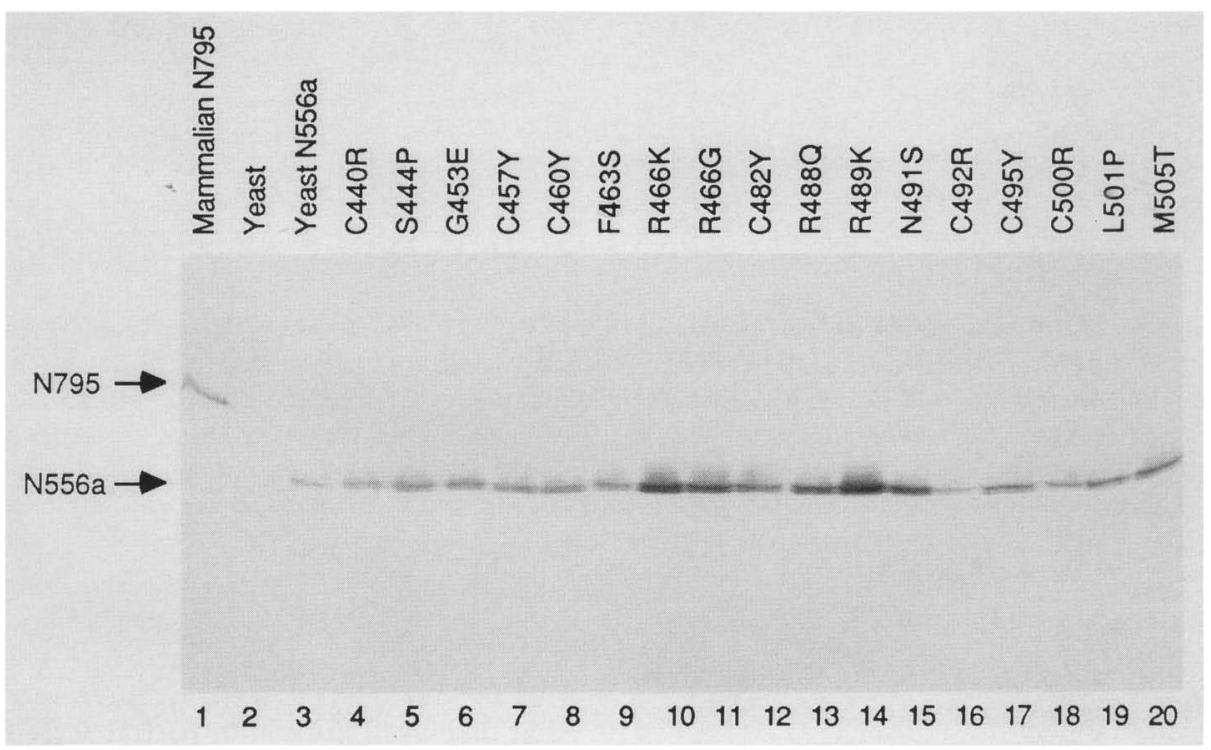

Figure 3. Stability of receptor finger domain mutants in yeast. Shown is an immunoblot of a mammalian extract and yeast extracts from the parental (BJ-G26.1) strain and from transformants producing various receptor derivatives; the mammalian N795 extract was prepared from HTC cell line 19G11.1 (Miesfeld et al. 1986). The extracts were fractionated by SDS-polyacrylamide gel electrophoresis and probed with a receptor-specific monoclonal antibody (Gametchu and Harrison 1984). Arrows indicate migration positions of the intact receptor $\mathrm{N} 795(88 \mathrm{kD})$ and the $\mathrm{N} 556$ constitutive receptor derivatives $(65 \mathrm{kD})$. 
Schena et al.

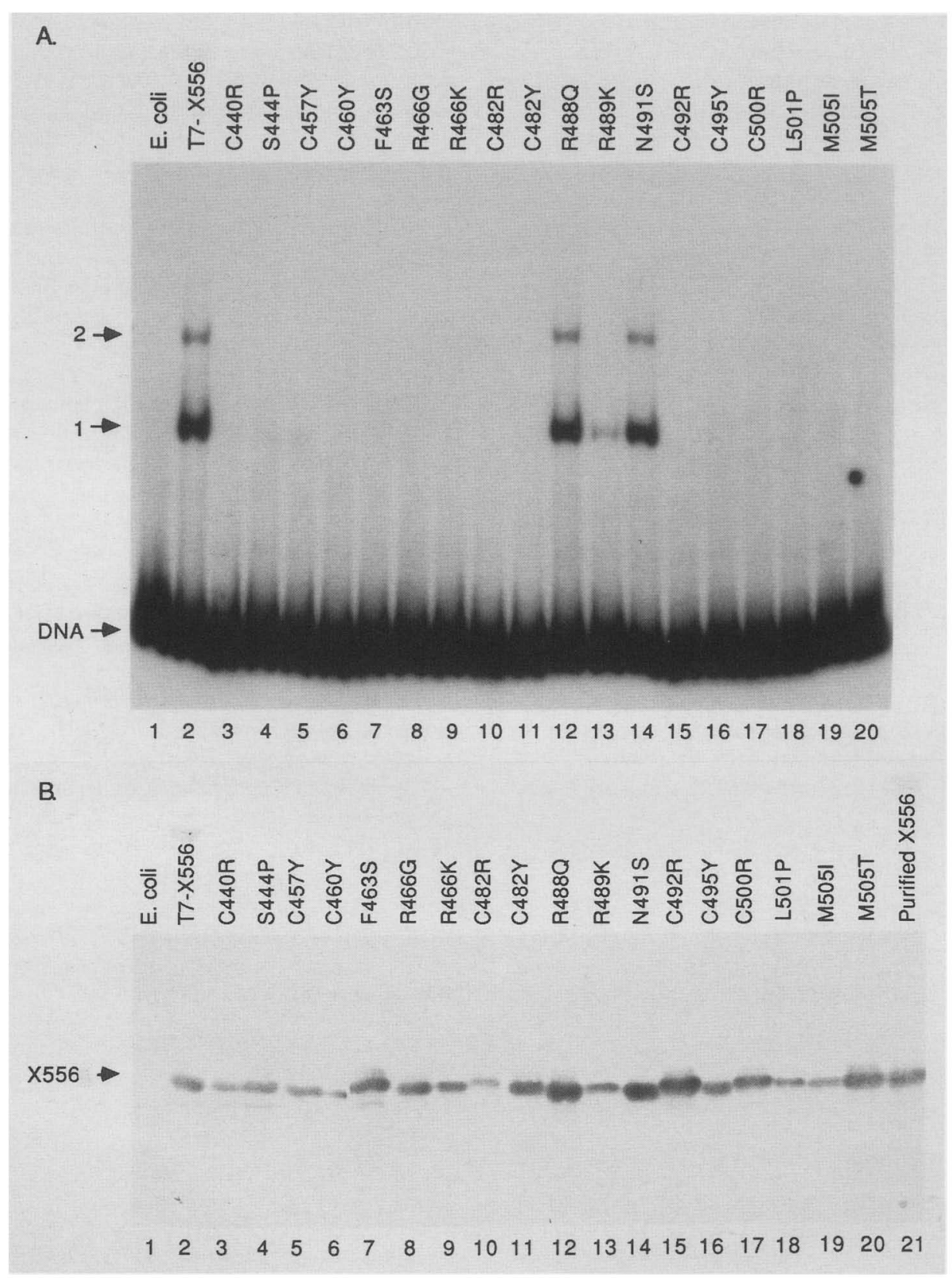

Figure 4. DNA-binding activities of the receptor finger domain in vitro. $(A)$ Mutant receptor species were assayed for DNA-binding in a gel mobility assay. E. coli extracts containing wild-type and mutant receptor derivatives were incubated with a labeled GRE-containing DNA fragment and electrophoresed in nondenaturing polyacrylamide gels, as described in Experimental procedures. Arrows indicate free DNA (DNA), and protein-DNA complexes with one (1) or two (2) molecules of receptor bound per molecule of DNA (J. LaBaer and K.R. Yamamoto, unpubl.). (B) E. coli extracts used for the gel mobility assay were fractionated by SDS-polyacrylamide gel electrophoresis and probed with a receptor-specific monoclonal antibody. The arrow indicates the mobility position of the X556a $(19-\mathrm{kD})$ receptor derivative. Relative receptor content in each extract was estimated from staining intensity, and minor differences were normalized prior to gel mobility analysis.

\section{A cold-sensitive receptor mutant}

Cold-sensitive mutations have commonly been associated with defects in protein-protein interactions (see Discussion). It seemed conceivable that some of the mutant receptors might be conditionally defective at elevated or reduced temperatures in yeast. Therefore, we replica-plated yeast strains expressing receptor mutants that displayed normal or partial activity at the standard $\left(30^{\circ} \mathrm{C}\right)$ temperature and assessed $\beta$-galactosidase activity on indicator plates at $19^{\circ} \mathrm{C}, 30^{\circ} \mathrm{C}$, and $37^{\circ} \mathrm{C}$. One mutant, $\mathrm{R} 489 \mathrm{~K}$, displayed a cold-sensitive phenotype; quantitation of $\beta$-galactosidase activity in liquid cultures confirmed that $\mathrm{R} 489 \mathrm{~K}$ was virtually inactive at $19^{\circ} \mathrm{C}$, 
whereas modest activity was observed at higher temperatures (Table 1). In contrast, the other receptors exhibited similar $\beta$-galactosidase induction levels at all three temperatures (Table 1; data not shown).

\section{Enhancement in mammalian cells}

Next we characterized the activities of the receptor mutants in animal cells. For these experiments, the mutant receptor inserts were transferred from yeast plasmids into mammalian expression vectors to reconstruct fulllength receptor derivatives. We tested each species for enhancement of a GRE-linked chloramphenicol acetyltransferase (CAT) reporter gene in transient cotransfections of CV-1 monkey cells, which lack endogenous receptor. Previous studies in both yeast and CV-1 cells established that the hormone-treated intact receptor (denoted N795) exhibits about twice the activity of the constitutive N556a derivative (see Table 2). Given the similar activities of these two receptor species in yeast and animal cells, we compared a series of mutants in these molecular and cellular backgrounds. As shown in Figure 5A, the wild-type receptor (N795) strongly enhanced CAT expression in a hormone-dependent manner, as did G453E, a neutral mutant in yeast (Fig. 5A, Table 2; see also Fig. 2B|. Indeed, six additional neutral mutants in yeast (L439P, E469K/Y474H, L475P R479K, G504E, G504R/ also displayed full activity in animal cells (data not shown). In contrast, most of the receptor mutants that failed to function in yeast were similarly inactive in CV-1 cells (Figs. 5A and 2B; C440R, S444P, C460Y, F463Y, C482Y, and C492R). Thus, despite the fact that the yeast and animal cell reporter plasmids employ different reporter genes, different promoters, and different GRE sequences (see Experimental procedures), the activities of receptor point mutants in yeast were closely paralleled in animal cells; this strongly supports the view that the receptor functions by a common mechanism in yeast and mammals.

Interestingly, relative enhancement in yeast and animal cells differed rather dramatically in four cases (Table 2). Of these four, R466K was somewhat difficult

Table 1. A cold-sensitive glucocorticoid receptor mutant

\begin{tabular}{lrrr}
\hline & \multicolumn{3}{c}{$\beta$-Galactosidase (units) } \\
\cline { 2 - 4 } Receptor & $19^{\circ} \mathrm{C}$ & $30^{\circ} \mathrm{C}$ & $37^{\circ} \mathrm{C}$ \\
\hline N556a & 433 & 496 & 554 \\
R489K & 3 & 34 & 42 \\
\hline
\end{tabular}

Yeast strain BJ-G26.1 was stably transformed with yeast expression plasmids encoding wild-type (N556a) or the R489K mutant receptor derivatives. Cultures were propagated at $19^{\circ} \mathrm{C}$, $30^{\circ} \mathrm{C}$ or $37^{\circ} \mathrm{C}$, and $\beta$-galactosidase activities were measured as described in Experimental procedures. A background activity of 2 units lobtained from a transformant lacking receptor sequences) was subtracted from the values shown, which represent the average of at least three independent measurements; individual determinations varied by $<20 \%$. No other cold-sensitive or temperature-sensitive phenotypes were detected among the 10 mutants that have been tested to date (data not shown).
Table 2. Enhancement by receptor mutants in yeast and mammalian cells

\begin{tabular}{|c|c|c|c|}
\hline \multirow[b]{2}{*}{ Receptor } & \multirow{2}{*}{$\begin{array}{l}\text { Yeast } \\
\text { (\% N556a) }\end{array}$} & \multicolumn{2}{|r|}{ CV-1 } \\
\hline & & $(\%$ N795) & (N556a normalized) \\
\hline N795 & 212 & 100 & 208 \\
\hline N556a & 100 & 48 & 100 \\
\hline G453E & 63 & 27 & 56 \\
\hline $\mathrm{R} 466 \mathrm{~K}$ & $<0.5$ & 3 & 6 \\
\hline $\mathrm{R} 488 \mathrm{Q}$ & 3 & 28 & 58 \\
\hline $\mathrm{R} 489 \mathrm{~K}$ & 12 & 44 & 92 \\
\hline N491S & 58 & $<0.5$ & $<0.5$ \\
\hline
\end{tabular}

Relative enhancement activities (normalized to N556a activity) of various receptor point mutants in yeast strain BJ-G26.1 were computed from $\beta$-galactosidase activities. Values represent the average of at least three independent assays that varied by $<20 \%$. Values from dexamethasone-treated mammalian $\mathrm{CV}-1$ cells were calculated from CAT activities of reporter gene GMCS normalized to the intact receptor N795. To facilitate comparison with the yeast data, the CV-1 results are also normalized to N556a; values represent the average of the three independent cotransfection experiments. Recent studies /data not shown/ confirm that the mutant phenotypes observed in yeast in the N556a backbone are unchanged when the same mutants are assayed in hormone-treated yeast cultures in an N795 backbone. The three mutants that display pc-like phenotypes in yeast or animal cells are R488Q, R489K, and N491S. Note that the DNA-binding activity of $\mathrm{R} 489 \mathrm{~K}$ is reduced in vitro and that in no case have we proved that the mutant proteins actually bind GREs in vivo. Such occupancy tests will be essential to assess the pc-like characteristics unequivocally.

to evaluate, as its activity is low in both cell types; it was inactive in yeast but displayed $6 \%$ of wild-type N556a activity in CV-1 cells (Table 2). More striking departures from parallel behavior in yeast and animal cells were observed with the other three mutants, all located in the tip of the second zinc finger. R489K displayed $12 \%$ of wild-type activity in yeast at $30^{\circ} \mathrm{C}$ and was cold sensitive, whereas it conferred $92 \%$ activity in animal cells; similarly, R488Q yielded $3 \%$ of full activity in yeast and $58 \%$ in animal cells (Figs. $2 \mathrm{~B}$ and $5 \mathrm{~A}$ ). In contrast, N491S was obtained in yeast as a neutral mutant, displaying $58 \%$ of full activity (Fig. $2 \mathrm{~B}$ ), and bound DNA normally in vitro (Fig. 4A), yet it conferred no detectable enhancement in mammalian cells (Fig. 5A). Conceivably, this cluster of mutations conferring nonparallel phenotypes in different species may identify a region of protein-protein contact (see Discussion).

\section{Repression in mammalian cells.}

The glucocorticoid receptor represses transcription by binding specifically at nGRE sequences (Sakai et al. 1988); the finger domain alone is sufficient to confer nGRE-mediated repression (Miesfeld et al. 1988). Therefore, we tested our point mutants for transcriptional repression by transient cotransfections of CV-1 cells with receptor expression plasmids, together with a reporter plasmid containing the bovine prolactin promoter and nGRE (Camper et al. 1985; Sakai et al. 1988). As with certain animal cell lines (Sakai et al. 1988), the prolactin 


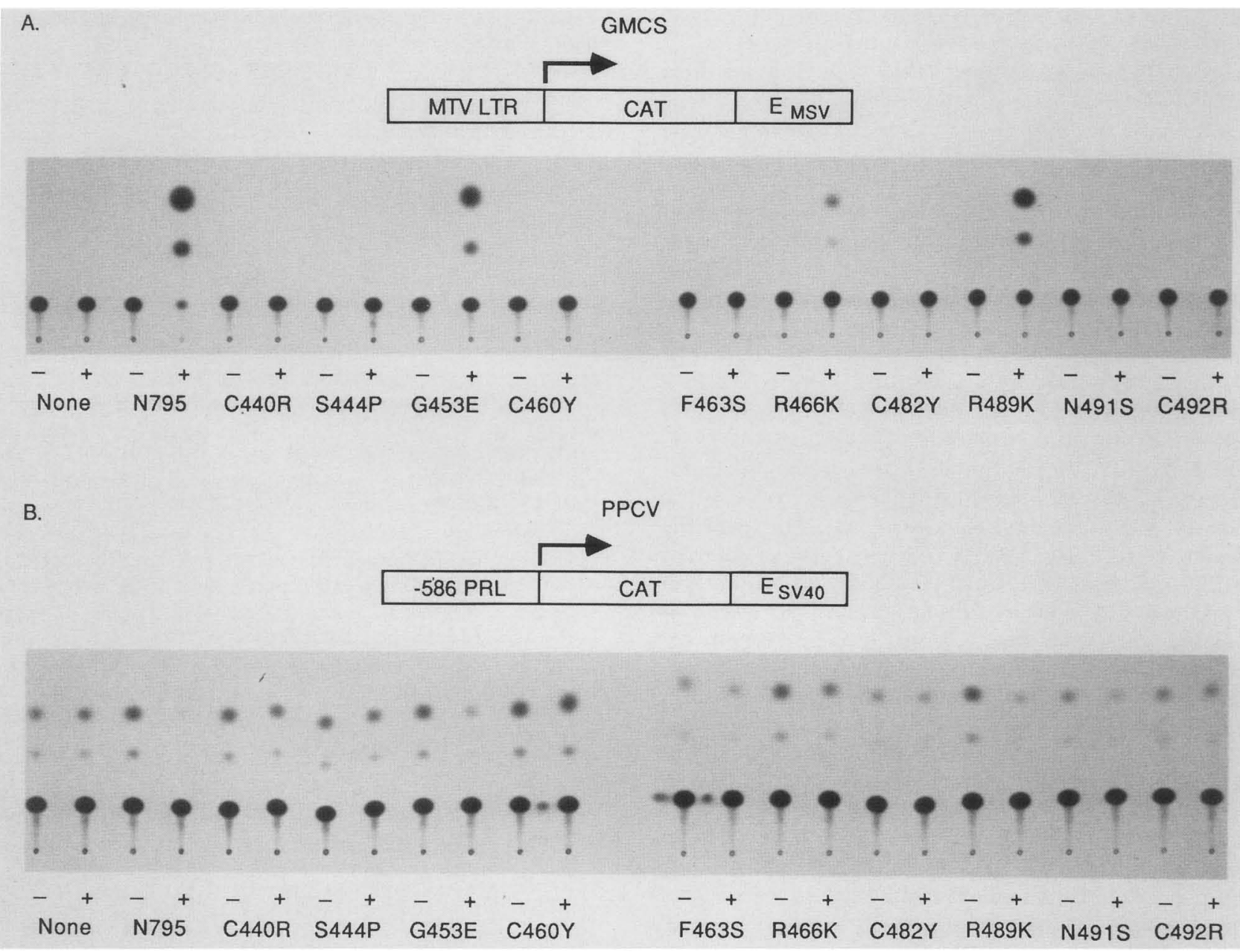

Figure 5. Enhancement and repression by receptor finger domain mutants in mammalian cells. $(A)$ Enhancement activities of the mutants were assayed in CV-1 cells. Reporter plasmid GMCS (DeFranco and Yamamoto 1986) contains the CAT gene driven by the MTV promoter and flanked by GREs from the MTV and MoMSV LTRs. Extracts were prepared from CV-1 cultures cotransfected transiently with GMCS DNA and with the intact receptor (N795) or with the mutant receptor derivatives shown, and propagated in the $(-)$ absence or $(+\mid$ presence of $0.1 \mu \mathrm{M}$ dexamethasone. Upper signals are the reaction products. $(B)$ Repression activities of mutants were assayed in CV-1 cells, as above, except that reporter plasmid PPCV was used in place of GMCS. PPCV contains the bovine prolactin nGREs and promoter (-586 PRL) driving the CAT gene fused upstream of the SV40 enhancer (Sakai et al. 1988).

nGRE is nonfunctional in yeast (data not shown). In CV-1 cells, however, hormone-dependent repression was observed with the wild-type receptor and with mutants that retained full or partial enhancement activity (Fig. 5B; see N795, G453E, and R489K). Conversely, mutants that failed to enhance transcription in yeast and were defective for DNA binding in vitro, lacked repression activity in animal cells (Fig. 5B, C440R, S444P, C460Y, F463S, C482Y, and C492R). Thus, many of the same amino acids that are essential for GRE binding and enhancement are also required for receptor-mediated repression at nGREs. Unexpectedly, two mutants, F463S and $\mathrm{C} 482 \mathrm{Y}$, produced lower basal activities (Fig. 5B); whether this indicates that these altered receptors can bind to nGREs, even in the absence of hormone, has not been tested directly. Finally, N491S, which bound DNA normally in vitro and enhanced transcription in yeast but not in animal cells, retained modest but reproducible repression activity at the prolactin nGRE (Fig. 5B).

\section{Discussion}

By coupling random mutagenesis in vitro and phenotypic screening in yeast, we recovered a novel series of point mutations in a 143-amino-acid (finger domain) segment of the rat glucocorticoid receptor. Characterization of these mutants in yeast, in animal cells and in vitro revealed that (1) point mutations that reduce enhancement activity are restricted to a 66-amino-acid subregion that encompasses the zinc fingers; (2) all of the mutant derivatives tested are soluble and accumulate to intracellular levels similar to the wild-type species; (3) in general (but see below), the mutants are phenotypically similar in yeast and animal cells, sup- 
porting further the notion that the receptor acts by a common mechanism in these diverse eukaryotes (Schena and Yamamoto 1988); (4) mutations that abolish GRE DNA binding are distributed across both fingers, especially at the cysteines thought to coordinate zinc ions, and in the five amino acids just downstream of each finger; (5) a series of mutations that selectively affect enhancement [positive control (pc)-like; see below] and a cold-sensitive mutation are tightly clustered in a portion of the second finger; (6) some of the mutants, particularly those with pc-like behavior, exhibit striking phenotypic differences in yeast and animal cells, consistent with the view that residues at these positions may be involved in protein-protein interactions.

It is worth noting that several transcription initiation factors and regulators from yeast and animal cells can function in cells from nonhomologous species (Buratowski et al. 1988; Chodosh et al. 1988; Kakidani and Ptashne 1988; Struhl 1988; Lambert et al. 1989); this implies that our strategy may provide a general approach for fine structure analysis of other gene products from organisms with complex or inaccessible genetics. The procedure appears particularly well suited to the facile isolation and preliminary characterization of a large number of mutations; indeed, the mutants described here were all obtained after treatment of only one strand of the DNA double helix with a single mutagen.

It is striking that every mutation that impaired receptor function was located in a 66-amino-acid segment coinciding precisely with the 61 residues of the zinc fingers plus five amino acids downstream of the second finger. Point mutations within this region were roughly equally distributed across the two fingers, showing clearly that each is essential for receptor function. Thus, our results strongly support the proposed zinc coordination pattern (Weinberger et al. 1985; Miesfeld et al. 1986) shown in Figure 2. We have not, however, ruled out an alternative scheme (Severne et al. 1988) suggesting that cysteine 492 may be involved in zinc binding, as mutation of this residue also abolished DNA binding. Direct biochemical and spectroscopic measurements comparing purified mutant and wild-type proteins will be necessary to determine unequivocally the correct coordination pattern. It is also notable that point mutations in the nuclear localization signal within the finger domain (residues 497-524) (Picard and Yamamoto 1987) were not recovered in our screen, implying that single amino acid changes in those sequences are insufficient to produce a phenotype.

Hollenberg and Evans (1988) employed a site-directed mutagenesis approach to this same region of the glucocorticoid receptor, substituting glycine residues for individual conserved amino acids within the two zinc finger motifs. At several positions, mutants that we isolated by screening in yeast were phenotypically similar to those observed by targeted mutagenesis of the same amino acids; moreover, we extend the conclusions of Hollenberg and Evans (1988) by establishing that the finger motifs are the essential functional sequences within the finger domain.

Mader et al. (1989) swapped segments of the estrogen and glucocorticoid receptors and constructed site-directed mutants to search for residues involved in distinguishing ERE and GRE DNA sequences; that study identified a cluster of three amino acids at the downstream side of the first finger that effect sequence specificity. Our results, in turn, emphasize the notion that both fingers in their entirety may be essential for forming a specific structure that facilitates sequence recognition by a small subset of amino acids.

The mutants that we analyzed were screened solely for defects in GRE-mediated positive regulation; in fact, we have been unable to demonstrate activity of the prolactin nGRE in yeast (M. Schena, unpubl.). We found that many of the resultant point mutants were severely deficient in GRE binding in vitro and that all of these DNA-binding mutants were also defective in nGRE-mediated repression when tested in CV-1 cells. Conversely, mutants that were competent to bind to GREs were also competent for repression via nGREs. Thus, although we have not identified residues that appear to distinguish GRE and nGRE sequences, our results support strongly the notion that DNA binding by the receptor is essential for repression. This conclusion disagrees with that of Adler et al. (1988), who studied estrogen and glucocorticoid inhibition of rat prolactin transcription and suggested from transient transfection experiments that repression is independent of the DNA-binding domains of the receptors. It may be relevant that Adler et al. (1988) used a recipient cell line that expresses endogenous estrogen and glucocorticoid receptors, perhaps resulting in competition or negative complementation between the wild-type endogenous and mutant transfected receptor derivatives.

The most interesting class of mutants that we obtained were those that distinguished sequences essential for transcriptional enhancement from those sequences involved in repression or DNA binding. This phenotypic class is reminiscent of the pc mutants of the $\lambda$ repressor, which fail to activate transcription while maintaining DNA-binding and repression activities (Guarente et al. 1982; Hochschild et al. 1983). The pc-type mutants that we isolated have three striking characteristics. First, they are tightly linked on the second finger (R488Q, R489K, N491S; Fig. 6; see also Figs. 2 and 5, and Table 2). Notably, Godowski et al. (1989) independently constructed a linker scanning mutation, LS-7, that displays a pc phenotype; remarkably, LS-7 is a double point mutant, P493R and A494S, in the same region of the second finger. Second, the only conditional mutant that we recovered is a cold-sensitive lesion that affects the severity of the R489K pc mutant; cold-sensitive mutants commonly reflect defects in protein-protein interactions (Guthrie et al. 1969; Jarvik and Botstein 1975). Third, only the three pc mutants exhibit strongly discordant phenotypes in yeast and animal cells, perhaps implying subtle differences in protein-protein contacts between receptor and homologous (but nonidentical) factors in yeast and animal cells. According to this view, such protein-protein interactions may be essential for receptormediated enhancement.

The cluster of pc mutants within the second finger ge- 

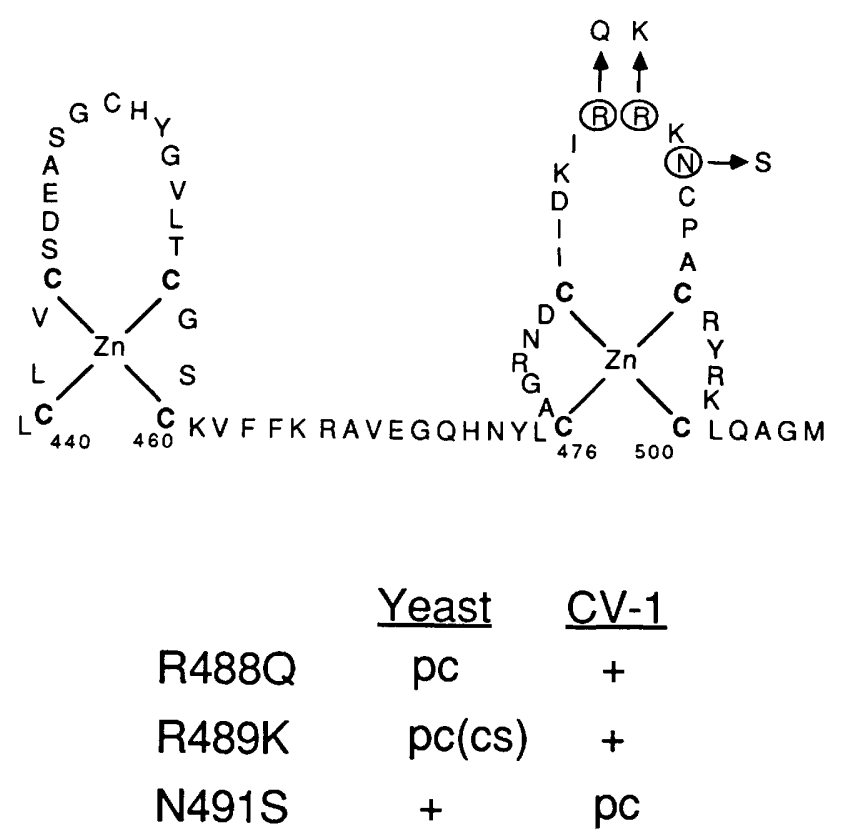

Figure 6. A tight cluster of receptor point mutations includes pc and cold-sensitive (cs) lesions and exhibits discordant phenotypes in yeast and animal cells. None of these three characteristics was associated with any other mutation isolated in the yeast screening procedure described here. Using linker scanning mutagenesis, Godowski et al. (1989) isolated another pc-like mutation that mapped to two amino acids in the same region (P493R and A494S) (for details, see text).

netically map an enhancement region (enh1) within the finger domain, extending the findings that this portion of the receptor alone is sufficient for enhancement, albeit with substantially reduced activity, both in vivo (Miesfeld et al. 1987) and in vitro (Freedman et al. 1989). In this regard, it is particularly intriguing that deletion mutagenesis (Giguere et al. 1986), as well as direct activity assays (Godowski et al. 1988), identified a second enhancement region, enh2, near the amino terminus of the receptor, and other work has inferred yet another distinct enhancement region within the receptor (Hollenberg and Evans 1988; see also Godowski et al. 1988). Because certain point mutations within enh1 can abolish enhancement by the otherwise wild-type receptor, multiple enhancement regions may be required in concert to enhance transcription, despite their capacities to function independently in certain contexts (Hollenberg et al. 1987; Miesfeld et al. 1987; Godowski et al. 1988). It may be possible to use mutations in enh1 to obtain suppressor mutants that identify factors with which enh1 interacts to give rise to specific regulation of gene transcription.

\section{Experimental procedures}

\section{Yeast strains and media}

The parent yeast strain BJ2168 (pep4-3, prc1-407, prb1-1122, ura3-52, trp-1, leu2) (Jones 1977; Sorger and Pelham 1987), carries defects in three protease genes. Strain BJ- G26.1 was constructed by integration of plasmid pl-G26.1, which contains a GRE-linked CYC1-lacZ fusion and URA3, at leu2 of BJ2163. Cultures were propagated in standard yeast media (Sherman et al. 1986).

\section{Plasmid constructions}

To facilitate mutagenesis of the receptor finger region, we constructed yeast shuttle plasmid pG-D, a pGPD-556a (Bitter and Egan 1984; Schena and Yamamoto 1988) derivative in which the pBR322 and the $2 \mu$-flanking sequences between BgIII and EcoRI were substituted with the pUC18 origin of replication and ampicillin resistance gene. The plasmid thus includes receptor sequences encoding residues $1-556$ driven by the yeast glycerol-3-phosphate dehydrogenase promoter, together with the yeast $2 \mu$ origin of replication and TRP1 gene.

The unique $X$ hoI site in $\mathrm{PG-D}$ at receptor amino acid 414 was introduced by oligonucleotide-directed mutagenesis (Kunkel et al. 1987). An ApaI-SstI fragment of the receptor cDNA (residues 317-556) was inserted into Bluescript M13 + (Messing 1983; Stratagene), and single-stranded DNA was isolated and hybridized (Schena 1989) to an oligonucleotide primer, 5'GGGTACTCGAGCCCTGG-3'; mismatch repair produced a conservative, single-base change in the serine codon at receptor amino acid 414 , thereby creating an $X h o I$ site. The ApaI-SstI receptor fragment then was reintroduced into the parent plasmid, yielding unique restriction sites flanking the receptor finger domain. The SstI site in pG-D is located six nucleotides downstream of receptor amino acid 556 in a polylinker that encodes 13 nonreceptor amino acids (GELEFPGLEDPST) prior to translation termination. Plasmid pl-G26.1, used to construct yeast strain BJ-G26.1, was made by digesting pHR35 (gift of R. Rothstein) with $\mathrm{NcOI}$ and BgIII in the URA3 gene and Tyl-17 element, respectively, and inserting an NcoI-BgIII fragment from pS-G26.1 (Schena and Yamamoto 1988) containing the URA3 gene and a GRE-linked CYC1-lacZ gene. Plasmid pl-G26.1 contains the yeast URA3 gene, a GRE-linked CYC1lacZ fusion, a 760-bp fragment of the LEU2 gene, and the pBR322 E. coli origin of replication and ampicillin resistance gene.

E. coli expression plasmid pT7X556 (Freedman et al. 1988) contains receptor residues 407-556 inserted downstream of the inducible bacteriophage $\mathrm{T} 7$ promoter. An XhoI site was generated at receptor residue 414 by subcloning a BamHI fragment encompassing receptor sequences into Bluescript $\mathrm{M13}+$ and performing oligonucleotide-directed mutagenesis (as above) to give pT7X556X. E. coli expression plasmids were constructed by shuttling XhoI-SstI mutant receptor inserts from yeast plasmids into phosphatase-treated pT7X556X digested with $X$ hoI and SstI.

To transfer the receptor point mutations into mammalian vectors, first we deleted wild-type receptor sequences from the expression vector (here denoted pVARO) used for N795 expression by Picard and Yamamoto (1987) by cleaving sites that encompass the receptor coding sequences at BamHI. Mutant receptor sequences were introduced by triple ligations of the BamHI-SphI (receptor residues 1-494) and SphI-BamHI (receptor residues 494-795) fragments into BamHI-digested, phosphatase treated pVARO. The final expression vectors contained the SV40 enhancer, the human $\alpha$-globin promoter driving the intact glucocorticoid receptor-coding region (residues $1-795)$, and the rabbit $\beta$-globin splice and poly(A) addition sites, cloned into SP64. Reporter plasmid GMCS (DeFranco and Yamamoto 1986) contains the mouse mammary tumor virus (MTV) long terminal repeat (LTR), the CAT gene, the SV40 poly(A) addition site, and the moloney murine sarcoma virus (MoMSV) enhancer, cloned into pSP64. Reporter 
plasmid PPCV (Miesfeld et al. 1988; Sakai et al. 1988) contains the bovine prolactin nGRE and promoter, the CAT gene, and the SV40 enhancer, cloned into pUC9.

\section{Chemical mutagenesis}

A fragment of yeast shuttle plasmid pG-D (encoding receptor residues 414-556) was excised using XhoI and SstI and inserted into $X$ hoI and SstI polylinker sites in Bluescript M13+. Singlestranded (sense strand) DNA (7 $\mu \mathrm{g}$ ) was treated for $20 \mathrm{~min}$ with sodium nitrite (Myers et al. 1985), and reverse transcriptase (BRL) was used to extend through the mutagenized region from a T7 primer hybridized to the Bluescript $\mathrm{M} 13+$ polylinker. Mutagenized, double-stranded receptor inserts were excised with XhoI and SstI, purified from low-melt agarose /Vogelstein and Gillespie 1979), and ligated to XhoI- and SstI-cleaved, unmutagenized, phosphatase-treated Bluescript M13 + DNA. The ligation mixture was transformed into $E$. coli and plasmid DNA was prepared from a pool of 104 bacterial transformants. The mutagenized receptor inserts were liberated with XhoI and SstI, purified from low-melt agarose, and inserted into phosphatasetreated pG-D digested with XhoI and SstI. Purified pG-D DNA was prepared from about $\sim 10^{4} \mathrm{E}$. coli transformants to give the mutagenized receptor pool (Fig. 1).

\section{Yeast screen and $\beta$-galactosidase assays}

Strain BJ-G26.1 was made competent with lithium acetate (Ito et al. 1983), and aliquots of $2 \times 10^{7}$ cells ( $2 \mathrm{OD}_{600}$ units) were transformed with $300 \mathrm{ng}$ of pG-D DNA from the mutagenized receptor pool. Transformants were selected on minimal plates deficient for uracil and tryptophan, and colonies $(\sim 300$ per plate) were transferred to nitrocellulose filters, and lysed in liquid nitrogen and scored for $\beta$-galactosidase production with $0.3 \mathrm{mg} / \mathrm{ml}$ of 5-bromo-4-chloro-indolyl- $\beta$-D-galactopyranoside (X-Gal); $\sim 0.4 \%$ of the colonies were white or light blue. Quantitative $\beta$-galactosidase measurements were made in triplicate on two separate occasions using yeast liquid cultures (Yocum et al. 1984); duplicate assays varied by $<20 \%$. $\beta$-Galactosidase units were defined as $10^{3}$ times the change in optical $\mathrm{OD}_{420}$ (due to hydrolysis of 0 -nitrophenyl- $\beta$-galactoside), divided by the product of the assay duration (min), times the culture volume $(\mathrm{ml})$, times $\mathrm{OD}_{600}$ of the culture.

\section{Yeast plasmid isolation and DNA sequencing}

Cells from $1.5 \mathrm{ml}$ of stationary yeast cultures were collected by centrifugation, washed in $1 \mathrm{ml}$ of $1 \mathrm{M}$ sorbitol, and incubated in $250 \mathrm{ml} 1 \mathrm{M}$ sorbitol, $50 \mathrm{mM}$ Tris (pH 7.5), $20 \mathrm{mM} \beta$-mercaptoethanol, and $2 \mathrm{mg} / \mathrm{ml}$ of zymolase $(20-\mathrm{T})$ at $37^{\circ} \mathrm{C}$ for $20 \mathrm{~min}$. Spheroplasts were pelleted, resuspended in $125 \mu$ l or $50 \mathrm{mM}$ EDTA and $0.3 \%$ SDS and incubated at $68^{\circ} \mathrm{C}$ for $20 \mathrm{~min}$. After removal of cell debris by ammonium acetate precipitation, plasmid DNA was phenol-extracted, ethanol-precipitated, and dissolved in $25 \mu \mathrm{l}$ of $10 \mathrm{mM}$ Tris- $\mathrm{HCl}(\mathrm{pH} 7.5), 1 \mathrm{mM}$ EDTA containing $100 \mu \mathrm{g} / \mathrm{ml}$ of RNase $A_{\text {; I }} \mu \mathrm{l}$ was used to transform $E$. coli strain DH5. Double-stranded minilysate DNA was subjected to DNA sequencing (Chen and Seeburg 1985), using oligonucleotide primers (Biomolecular Resource Center, UCSF) to receptor sequences encompassing the mutagenized region (amino acids 414-556).

\section{DNA binding}

The XhoI-SstI receptor segments from various mutants were shuttled into the corresponding sites of a T7X556a expression plasmid, in which the receptor sequences are expressed from a bacteriophage T7 promoter; E. coli transformants were induced to overexpress the wild-type or mutant derivatives, as described previously (Freedman et al. 1988). The receptor species were precipitated from $30 \%$ ammonium sulfate (Freedman et al. 1988 ) and were redissolved in TEGDZ50 buffer [ $50 \mathrm{~mm}$ Tris$\mathrm{HCl}\{\mathrm{pH} 7.5\}, 0.5 \mathrm{~mm}$ EDTA, $10 \%$ glycerol, $2.5 \mathrm{mM} \mathrm{DTT}, 50 \mu \mathrm{M}$ $\mathrm{ZnCl}_{2}, 50 \mathrm{mM} \mathrm{NaCl}$ ] and dialyzed against the same buffer; extract protein concentrations were $\sim 10 \mathrm{mg} / \mathrm{ml}$, with the receptor derivative comprising $\sim 10 \%$ of the total protein. Gel mobility assays (Fried and Crothers 1981) were performed by preincubating $40 \mathrm{ng}$ of extract protein with $1 \mu \mathrm{g}$ of poly[d(I-C)] for $10 \mathrm{~min}$ at room temperature, followed by addition of a ${ }^{32} \mathrm{P}$ labeled 256-bp fragment containing a 27-bp GRE (J. La Baer and K.R. Yamamoto, unpubl.); receptor protein-DNA complexes were separated from free DNA on a nondenaturing $7.8 \%$ polyacrylamide gel at $4^{\circ} \mathrm{C}$.

\section{Cell culture and DNA transfection}

CV-1 cells were propagated in Dulbecco's modified Eagle's medium (Cell Culture Facility, UCSF), supplemented with $5 \%$ defined calf serum (HyClone). Mixtures of pVARO expression vectors $(2 \mu \mathrm{g})$ and GMCS or PPCV reporter plasmids $(0.5 \mu \mathrm{g})$ were cotransfected (Graham and van der Eb 1973) into subconfluent cultures of CV-1 cells in $60-\mathrm{mm}$ dishes. Cells were incubated with the calcium phosphate precipitate for $16 \mathrm{hr}$ and transferred to fresh medium with or without $0.2 \mu \mathrm{M}$ dexamethasone for an additional $24 \mathrm{hr}$. Extracts were prepared by three rounds of freezing $\left(-70^{\circ} \mathrm{C}\right)$ and thawing $\left(68^{\circ} \mathrm{C}\right)$, followed by centrifugation at $15,000 \mathrm{~g}$ for $15 \mathrm{~min}$. The CAT activity in $7 \mu \mathrm{g}$ of soluble protein from each extract was determined as described (Gorman et al. 1982); extracts prepared from cells transfected with GMCS or PPCV were incubated with substrate at $37^{\circ} \mathrm{C}$ for $5 \mathrm{hr}$ or $20 \mathrm{hr}$, respectively.

\section{Acknowledgments}

We are indebted to Bob Harrison for monoclonal antibody BUGR2, Rodney Rothstein and Hans Ronne for plasmid pHR35, Hugh Pelham for yeast BJ2168, and Rick Myers for help with the chemical mutagenesis. We also thank Sandy Johnson, Rick Myers, and Didier Picard for comments on the manuscript, and Kathy Mulherin and Bonnie Maler for expert preparation of text and figures, respectively. This work was supported by grants from the National Institutes of Health and the National Science Foundation. L.P.F. was a Bank of AmericaGiannini Foundation fellow.

\section{References}

Adler, S., M.L. Waterman, X. He, and M.G. Rosenfeld. 1988. Steroid receptor-mediated inhibition of rat prolactin gene expression does not require the receptor DNA-binding domain. Cell 52: 685-695.

Beato, M. 1989. Gene regulation by steroid hormones. Cell 56: $335-344$.

Berg, J. M. 1986. Nucleic acid-binding proteins: More metalbinding fingers. Nature 319: 264-265.

Bitter, G.A. and K.M. Egan. 1984. Expression of heterologous genes in $S$. cerevisiae from vectors utilizing the glyceraldehyde-3-phosphate dehydrogenase gene promoter. Gene 32: $263-269$.

Buratowski, S., S. Hahn, P.A. Sharp, and L. Guarente. 1988. Function of a yeast TATA element-binding protein in a mammalian transcription system. Nature 334: 37-42.

Camper, S.A., Y.A.S. Yao, and F.M. Rottman. 1985. Hormonal regulation of the bovine prolactin promoter in rat pituitary 
tumor cells. J. Biol. Chem. 260: 12246-12251.

Chandler, V.L., B.A. Maler and K.R. Yamamoto. 1983. DNA sequences bound specifically by glucocorticoid receptor in vitro render a heterologous promoter hormone responsive in vivo. Cell 33: 489-499.

Chen, E.Y. and P.H. Seeburg. 1985. A fast and simple method for sequencing plasmid DNA. DNA 4: 165-170.

Chodosh, L.A., J. Olesen, S. Hahn, A.S. Baldwin, L. Guarente, and P.A. Sharp. 1988. A yeast and a human CCAAT-binding protein have heterologous subunits that are functionally interchangeable. Cell 53: 25-35.

DeFranco, D. and K.R. Yamamoto 1986. Two different factors act separately or together to specify functionally distinct activities at a single transcriptional enhancer. Mol. Cell. Biol. 6: $993-1001$.

Evans, R.M. 1988. The steroid and thyroid hormone receptor superfamily. Science 240: 889-895.

Freedman, L.P., S.K. Yoshinaga, J.N. Vanderbilt and K.R. Yamamoto. 1989. In vitro transcriptional enhancement by purified derivatives of the glucocorticoid receptor. Science 245: 298-301.

Freedman, L.P., B.F. Luisi, Z.R. Korszun, R. Basavappa, P.B. Sigler, and K.R. Yamamoto 1988. The function and structure of the metal coordination sites within the glucocorticoid receptor DNA-binding domain. Nature 334: 543-546.

Fried, M.G. and D.M. Crothers. 1981. Equilibria and kinetics of lac repressor-operator interactions by polyacrylamide gel electrophoresis. Nucleic Acids Res. 9: 6505-6525.

Gametchu, B. and R.W. Harrison. 1984. Characterization of a monoclonal antibody to the rat liver glucocorticoid receptor. Endocrinology 114: 274-279.

Giguere, V., S.M. Hollenberg, M.G. Rosenfeld, and R.M. Evans. 1986. Functional domains of the human glucocorticoid receptor. Cell 46: 645-652.

Godowski, P.J., D. Picard, and K.R. Yamamoto. 1988. Signal transduction and transcriptional regulation by glucocorticoid receptor-LexA fusion proteins. Science 241: 812-816.

Godowski, P.J., D.D. Sakai, and K.R. Yamamoto. 1989. Signal transduction and transcriptional regulation by the glucocorticoid receptor. In DNA-protein interactions in transcription, UCLA Symposium on Molecular and Cellular Biology, new series, (ed. J. Gralla). vol. 95, pp. 197-210. Alan R. Liss, New York.

Godowski, P.J., S. Rusconi, R. Miesfeld, and K.R. Yamamoto. 1987. Glucocorticoid receptor mutants that are constitutive activators of transcriptional enhancement. Nature 325: 365-368.

Gorman, C.M., L.F. Moffat, and B.H. Howard. 1982. Recombinant genomes which express chloramphenicol acetyltransferase in mammalian cells. Mol. Cell. Biol. 2: 1044-1051.

Graham, F.L. and A.J. van der Eb. 1973. A new technique for the assay of infectivity of human adenovirus 5 DNA. Virology 52: $456-467$

Green, S. and P. Chambon. 1988. Nuclear receptors enhance our understanding of transcription regulation. Trends Genet. 4: 309-314.

Guarente, L. and E. Hoar. 1984. Upstream activation sites of the $\mathrm{CYCl}$ gene of S. cerevisiae are active when inverted but not when placed downstream of the TATA box. Proc. Natl. Acad. Sci. 81: 7860-7864.

Guarente, L., J.S. Nye, A. Hochschild, and M. Ptashne. 1982. A mutant $\lambda$ repressor with a specific defect in its positive control function. Proc. Natl. Acad. Sci. 73: 2249-2253.

Guthrie, C., H. Nashim, and M. Nomura. 1969. Structure and function of $\mathrm{E}$. coli ribosomes: cold sensitive mutants defective in ribosome assembly. Proc. Natl. Acad. Sci. 63: 384-
391.

Hochschild, A., N. Irwin, and M. Ptashne. 1983. Repressor structure and the mechanism of positive control. Cell 32: 319-325.

Hollenberg, S.M. and R.M. Evans. 1988. Multiple and cooperative trans-activation domains of the human glucocorticoid receptor. Cell 55: 899-906.

Hollenberg, S.M., V. Giguere, P. Sequi, and R.M. Evans. 1987. Colocalization of DNA-binding and transcriptional activation functions in the human glucocorticoid receptor. Cell 49: $39-46$.

Hope, I.A. and K. Struhl. 1986. Functional dissection of a eukaryotic transcriptional activator protein, GCN4 of yeast. Cell 46: 885-894.

Ito, H., Y. Fukada, K. Mureata, and A. Kimura. 1983. Transformation of intact yeast cells treated with alkali cations. $/$. Bacteriol. 153: 163-168.

Jarvik, J. and D. Botstein. 1975. Conditional-lethal mutations that suppress genetic defects in morphogenesis by altering structural proteins. Proc. Natl. Acad. Sci. 72: 2738-2742.

Jones, E.W. 1977. Proteinase mutants of Saccharomyces cerevisiae. Genetics 85: 23-33.

Kakidani, H. and M. Ptashne. 1988. Gal4 activates gene expression in mammalian cells. Cell 52: 161-167.

Kunkel, T.A., J.D. Roberts, and R.A. Zakour. 1987. Rapid and efficient site-specific mutagenesis without phenotype selection. Methods Enzymol. 154: 367-382.

Lambert, P.F., N. Dostatni, A.A. McBride, M. Yaniv, P.M. Howley, and B. Arcangioli. 1989. Functional analysis of the papilloma virus E2 trans-activator in Saccharomyces cerevisiae. Genes Dev. 3: 38-48.

Lech, K., K. Anderson, and R. Brent. 1988. DNA-bound Fos proteins activate transcription in yeast. Cell 52: 179-184.

Ma, J. and M. Ptashne. 1987. Deletion analysis of Gal4 defines two trancriptional activating segments. Cell 48: 847-853.

Mader, S., V. Jumar, H. de Verneuil, and P. Chambon. 1989. Three amino acids of the estrogen receptor are essential to its ability to distinguish an estrogen from a glucocorticoid responsive element. Nature 338: 271-274.

Messing, J. 1983 . New M13 vectors for cloning. Methods Enzymol. 101: 20-78.

Metzger, D., H.H. White, and P. Chambon. 1988. The human estrogen receptor functions in yeast. Nature 334: 31-36.

Miesfeld, R., P.J. Godowski, B.A. Maler, and K.R. Yamamoto. 1987. Glucocorticoid receptor mutants that define a small region sufficient for enhancer activation. Science 236: $423-$ 427.

Miesfeld, R., D. Sakai, A. Inoue, M. Schena, P.J. Godowski, and K.R. Yamamoto. 1988. Glucocorticoid receptor sequences that confer positive and negative transcriptional regulation. In Steroid hormone action, UCLA Symposium on Molecular and Cellular Biology, (ed. G. Ringold). pp. 193-200. Alan R. Liss, New York.

Miesfeld, R., S. Rusconi, P.J. Godowski, B.A. Maler, S. Okret, A.-C. Wikstrom, J.-A. Gustafsson, and K.R. Yamamoto. 1986. Genetic complementation of a glucocorticoid receptor deficiency by expression of cloned receptor cDNA. Cell 46: 389-399.

Miller, J., A.D. McLachland, and A. Klug. 1985. Repetitive zinc-binding domains in the protein transcription factor IIIA from Xenopus oocytes. EMBO I. 4: 1609-1614.

Mordacq, J.C. and D.I.H. Linzer. 1989. Co-localization of elements required for phorbol ester stimulation and glucocorticoid repression of proliferin gene expression. Genes Dev. 3: 760-769.

Myers, R.M., L.S. Lerman, and T. Maniatis. 1985. A general 
method for saturation mutagenesis of cloned DNA fragments. Science 229: 242-247.

Payvar, F., D. DeFranco, G.L. Firestone, B. Edgar, O. Wrange, S. Okret, J.-Å. Gustafsson, and K.R. Yamamoto. 1983. Sequence-specific binding of glucocorticoid receptor to MTV DNA at sites within and upstream of the transcribed region. Cell 35: 381-392.

Picard, D. and K.R. Yamamoto. 1987. Two signals mediate hormone-dependent nuclear localization of the glucocorticoid receptor. $E M B O$ I. 6: 3333-3340.

Ptashne, M. 1986. Gene regulation by proteins acting nearby and at a distance. Nature 322: 697-701.

. 1988. How eukaryotic transcriptional activators work. Nature 335: 683-689.

Rusconi, S. and K.R. Yamamoto. 1987. Functional dissection of the hormone and DNA-binding activities of the glucocorticoid receptor. EMBO J. 6: 1309-1315.

Sakai, D.D., S. Helms, J. Carlstedt-Duke, J.-Å. Gustafsson, F.M. Rottman, and K.R. Yamamoto. 1988. Hormone-mediated repression of transcription: A negative glucocorticoid response element from the bovine prolactin gene. Genes Dev. 2: 1144-1154.

Scheidereit, C., S. Geisse, H.M. Westphal, and M. Beato. 1983. The glucocorticoid receptor binds to defined nucleotide sequences near the promoter of mouse mammary tumor virus. Nature 304: 749-752.

Schena, M. 1989. High efficiency olignucleotide-directed mutagenesis using sequenase. Comments 15-2: 23.

Schena, M. and K.R. Yamamoto. 1988. Mammalian glucocorticoid receptor derivatives enhance transcription in yeast. Science 241: 965-967.

Severne, Y., S. Wieland, W. Schaffner, and S. Rusconi. 1988. Metal binding 'finger' structures in the glucocorticoid receptor defined by site-directed mutagenesis. EMBO $J$. 7: 2503-2508.

Sherman, F., G. Fink, and J.B. Hicks. 1986. Methods in yeast genetics. Cold Spring Harbor Laboratory, Cold Spring Harbor, New York.

Sorger, P.K. and H.R.B. Pelham. 1987. Purification and characterization of a heat-shock element binding protein from yeast. $E M B O$ I. 6: 3035-3041.

Struhl, K. 1988. The Jun oncoprotein, a vertebrate transcription factor, activates transcription in yeast. Nature 332: 649651.

Studier, F.W. and B.A. Moffat. 1986. Use of bacteriophage T7 RNA polymerase to direct selective high-level expression of cloned genes. J. Mol. Biol. 189: 113-130.

Vogelstein, B. and D. Gillespie. 1979. Preparative and analytical purification of DNA fragments from agarose. Proc. Natl. Acad. Sci. 76: 615-619.

Webster, N., J.R. Jin, S. Green, M. Hollis, and P. Chambon. 1988. The yeast $\mathrm{UAS}_{\mathrm{G}}$ is a transcriptional enhancer in human HeLa cells in the presence of the Gal4 trans-activator. Cell 52: 169-178.

Weinberger, C., S.M. Hollenberger, M.G. Rosenfeld, and R.M Evans. 1985. Domain structure of human glucocorticoid receptor and its relationship to the v-erb-A oncogene product. Nature 318: 670-672.

Yamamoto K.R. 1985. Steroid receptor regulated transcription of specific genes and gene networks. Annu. Rev. Genet. 19: 209-252.

1989. A conceptual view of transcriptional regulation. Am. Zool. 29: 537-547.

Yocum, R.R., S. Hanley, R.W. West, Jr., and M. Ptashne. 1984. Use of $l a c Z$ fusions to delimit regulatory elements of inducible divergent Gall-Gallo promoter in S. cerevisiae. Mol. Cell Biol. 4: 1985-1998. 


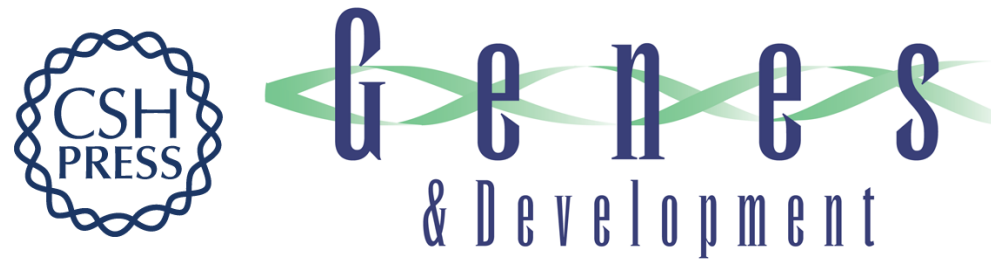

\section{Mutations in the glucocorticoid receptor zinc finger region that distinguish interdigitated DNA binding and transcriptional enhancement activities.}

M Schena, L P Freedman and K R Yamamoto

Genes Dev. 1989, 3:

Access the most recent version at doi:10.1101/gad.3.10.1590

References This article cites 63 articles, 19 of which can be accessed free at:

http://genesdev.cshlp.org/content/3/10/1590.full.html\#ref-list-1

License

Email Alerting

Service

Receive free email alerts when new articles cite this article - sign up in the box at the top right corner of the article or click here.

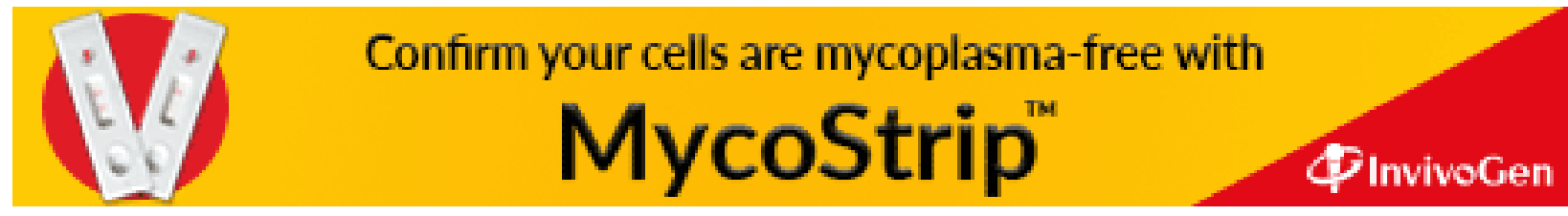

